PROCEEDINGS OF THE

AMERICAN MATHEMATICAL SOCIETY

Volume 126, Number 10, October 1998, Pages 2863-2871

S 0002-9939(98)04351-2

\title{
HIGHER-DIMENSIONAL AHLFORS-BEURLING TYPE INEQUALITIES IN CLIFFORD ANALYSIS
}

\author{
MIRCEA MARTIN
}

(Communicated by Palle E. T. Jorgensen)

\begin{abstract}
A generalization to higher dimensions of a classical inequality due to Ahlfors and Buerling is proved. As a consequence, an extension of Alexander's quantitative version of Hartogs-Rosenthal Theorem is derived. Both results are stated and proved within the framework of Clifford analysis.
\end{abstract}

\section{INTRODUCTION}

The theme of this article goes back to an inequality proved by Ahlfors and Beurling $[\mathrm{AB}]$, which states that

$$
\left|\frac{1}{2 \pi} \int_{X} \frac{\mathrm{d} \lambda}{\zeta-a}\right| \leq\left[\frac{1}{4 \pi} \cdot \lambda(X)\right]^{1 / 2}, \quad a \in \mathbb{C},
$$

where $X$ is a compact subset of the complex plane $\mathbb{C}$ and $\lambda$ is the two-dimensional Lebesgue measure. This inequality supplies an important tool in studying rational approximation (see, for instance, $[\mathrm{G}]$ or $[\mathrm{B}]$ ). A striking consequence of (1.1) has been singled out by Alexander [A1], [A2]. For $X$ as above, we let $C(X)$ and $R(X)$ denote the Banach algebra of complex-valued continuous functions on $X$ and the uniform closure in $C(X)$ of the rational functions analytic on open neighborhoods of $X$, respectively. Alexander's inequality asserts that

$$
\operatorname{dist}_{C(X)}[\bar{\zeta}, R(X)] \leq\left[\frac{1}{\pi} \cdot \lambda(X)\right]^{1 / 2},
$$

where $\bar{\zeta}$ stands for the conjugate of the complex coordinate function on $\mathbb{C}$. If $X$ has area zero, then $\bar{\zeta} \in R(X)$, and by the Stone-Weierstrass Theorem one concludes that $R(X)=C(X)$, so (1.2) implies the classical Hartogs-Rosenthal Theorem.

Another nice feature of Alexander's inequality (1.2) has been revealed by Axler and Shapiro [AS]. To make a point we first recall an inequality for seminormal Hilbert space operators due to Putnam $[\mathrm{Pu}]$. Specifically, suppose that $T$ is a Hilbert space operator such that $T^{*} T-T T^{*}$ is semidefinite, and let $\operatorname{spec}(T)$ denote the spectrum of $T$. Putnam's Theorem amounts to the inequality

$$
\left\|T^{*} T-T T^{*}\right\| \leq \frac{1}{\pi} \lambda(\operatorname{spec}(T))
$$

Received by the editors February 18, 1997.

1991 Mathematics Subject Classification. Primary 31B10, 41A20, 41A63.

Key words and phrases. Clifford analysis, approximation theory.

This work was supported in part by NSF Grant DMS-9301187.

(C) 1998 American Mathematical Society 
Axler and Shapiro pointed out that Putnam's inequality (1.3) for subnormal operators can be proved based on - in fact it is equivalent to - inequality (1.2).

The relationship between Ahlfors-Beurling inequality (1.1) and the theory of seminormal operators is in fact deeper than the few comments above might suggest. In this regard we should mention a generalization of (1.1) proved by Putinar $[\mathrm{P}]$ by relying on some subtle structural properties of hyponormal operators with a one-dimensional self-commutator. Putinar's result states that

$$
\left|\frac{1}{2 \pi} \int_{\mathbb{C}} \frac{\varphi(\zeta)}{\zeta-a} \mathrm{~d} \lambda\right| \leq \frac{1}{2 \sqrt{\pi}}\|\varphi\|_{1}^{1 / 2}\|\varphi\|_{\infty}^{1 / 2},
$$

for any non-negative function $\varphi \in L^{1}(\mathbb{C}) \cap L^{\infty}(\mathbb{C})$. For some other applications of the theory of hyponormal operators to function theory we refer the reader to the two already cited articles [AS] and [P], and to the survey paper [VPY].

The main goal of our article is to extend the Ahlfors-Beurling inequality (1.1) to higher dimensions. Along the way we will also derive multivariable substitutes for (1.2) and (1.4). As a highlight of our approach we notice that all the basic devices we will be dealing with are borrowed from Clifford analysis. Excellent accounts on the subject can be found in [BDS], [DSS], and [GM].

The only inequality listed above but completely ignored in the sequel is Putnam's inequality (1.3). We intend to make up for this loss elsewhere, in connection with the theory of seminormal tuples of Hilbert space operators.

\section{Uniform estimates of higher-Dimensional CAUChy transforms}

In this section we generalize both inequalities (1.1) and (1.4) to higher dimensions by means of an appropriate substitute for the classical Cauchy kernel.

2.1. Throughout our article we will let $\mathfrak{A}_{m}$ denote the real Clifford algebra associated with the Euclidean space $\mathbb{R}^{m}, m \geq 1$. The algebra $\mathfrak{A}_{m}$ can be described in terms of generators and relations as the unital associative real algebra generated by the standard orthonormal basis $\left\{e_{1}, e_{2}, \ldots, e_{m}\right\}$ for $\mathbb{R}^{m}$, subject to the relations

$$
e_{j} e_{j}+e_{j} e_{i}=-2 \delta_{i j} e_{0}, \quad 1 \leq i, j \leq m,
$$

where $e_{0}$ stands for the identity of $\mathfrak{A}_{m}$, and $\delta_{i j}$ equals 1 or -1 as $i=j$ or $i \neq j$. The dimension of $\mathfrak{A}_{m}$ as a real vector space equals $2^{m}$, and the set consisting of $e_{0}$ and all reduced products

$$
e_{I}=e_{i_{1}} \cdots e_{i_{p}}, \quad I=\left(i_{1}, \ldots, i_{p}\right), \quad 1 \leq i_{1}<\cdots<i_{p} \leq m,
$$

yields a basis for $\mathfrak{A}_{m}$. The Clifford algebra $\mathfrak{A}_{m}$ comes in with a rich structure. First, $\mathfrak{A}_{m}$ has an inner product, such that the basis just defined is orthonormal. The corresponding norm will be denoted by $|\cdot|$. Further, by regarding $\mathfrak{A}_{m}$ as an algebra of operators on itself, we convert $\mathfrak{A}_{m}$ into a real $C^{*}$-algebra. The $C^{*}$ algebra norm on $\mathfrak{A}_{m}$ is denoted by $\|\cdot\|$. The involution on $\mathfrak{A}_{m}$ is provided by Clifford conjugation, which is uniquely determined by the rule

$$
\bar{e}_{i}=-e_{i}, \quad 1 \leq i \leq m .
$$

We next identify any $x=\left(x_{0}, x_{1}, \ldots, x_{m}\right)$ in $\mathbb{R}^{m+1}$ with the element $x=x_{0} e_{0}+$ $x_{1} e_{1}+\cdots+x_{m} e_{m}$ of $\mathfrak{A}_{m}$, and thus we get an embedding of $\mathbb{R}^{m+1}$ into $\mathfrak{A}_{m}$. The two norms $|\cdot|$ and $\|\cdot\|$ on $\mathfrak{A}_{m}$ defined above induce the Euclidean norm on $\mathbb{R}^{m+1}$. 
2.2. For a later use, we let $\mathfrak{H}$ denote a unitary left $\mathfrak{A}_{m}$-module, that is, a finitedimensional Hilbert space upon which the algebra $\mathfrak{A}_{m}$ acts on the left such that every generator $e_{i}$ of $\mathfrak{A}_{m}, 1 \leq i \leq m$, determines a skew-adjoint unitary operator, and $e_{0}$ corresponds to the identity operator. Assume next that $\Omega \subseteq \mathbb{R}^{m+1}$ is an open set. The space $C^{\infty}(\Omega, \mathfrak{H})$ of smooth $\mathfrak{H}$-valued functions on $\Omega$ is a left $\mathfrak{A}_{m^{-}}$ module under pointwise multiplication. Therefore, it makes sense to define a linear first-order differential operator $D: C^{\infty}(\Omega, \mathfrak{H}) \rightarrow C^{\infty}(\Omega, \mathfrak{H})$ by setting

$$
D=e_{0} \partial_{0}+e_{1} \partial_{1}+\cdots+e_{m} \partial_{m},
$$

where $\partial_{k}=\partial / \partial x_{k}, 0 \leq k \leq m$. The so defined operator $D$ is called the Euclidean Dirac operator on $C^{\infty}(\Omega, \mathfrak{H})$. As another piece of notion and terminology, we set

$$
\mathfrak{M}(\Omega, \mathfrak{H})=\left\{\varphi \in C^{\infty}(\Omega, \mathfrak{H}): D \varphi=0\right\},
$$

and refer to any function $\varphi$ in $\mathfrak{M}(\Omega, \mathfrak{H})$ as a monogenic - or Clifford analytic- $\mathfrak{H}$ valued function on $\Omega$ (see [BDS], [DSS], or [GM]).

2.3. A basic example of a monogenic $\mathfrak{A}_{m}$-valued function is provided by the Cauchy kernel $E$ on $\mathbb{R}^{m+1}$ defined as

$$
E(x)=\frac{1}{\sigma_{m}} \cdot \frac{\bar{x}}{|x|^{m+1}}, \quad x \in \mathbb{R}_{0}^{m+1}=\mathbb{R}^{m+1} \backslash\{0\},
$$

where $\sigma_{m}$ is the area of the unit sphere $\mathbb{S}^{m}$ in $\mathbb{R}^{m+1}$, and $\bar{x}$ and $|x|$ stand for the Clifford conjugate and the Euclidean norm of a vector $x$ in $\mathbb{R}^{m+1}$, respectively. Its important role in Clifford analysis is illustrated by the following construction.

Given $\Omega$ and $\mathfrak{H}$ as in (2.1), suppose that $\Delta$ is a bounded open subset of $\Omega$ with a smooth boundary $\partial \Delta$, and let $\eta(x) \in \mathbb{R}^{m+1} \subseteq \mathfrak{A}_{m}$ be the inward pointing unit normal to $\partial \Delta$ at $x \in \partial \Delta$. To every function $\varphi \in C^{\infty}(\Omega, \mathfrak{H})$ one associates two $\mathfrak{H}$-valued smooth functions $\mathcal{C}_{\partial \Delta} \varphi$ and $\mathcal{C}_{\Delta} \varphi$ on $\Delta$ by setting

$$
\mathcal{C}_{\partial \Delta} \varphi(a)=\int_{\partial \Delta} E(a-x) \eta(x) \varphi(x) \mathrm{d} \sigma(x), \quad a \in \Delta,
$$

and

$$
\mathcal{C}_{\Delta} \varphi(a)=\int_{\Delta} E(a-x) D \varphi(x) \mathrm{d} \lambda(x), \quad a \in \Delta,
$$

where $\sigma$ stands for the surface area measure on $\partial \Delta$ and $\lambda$ is the Lebesgue measure on $\mathbb{R}^{m+1}$. The two so defined functions are related to the original function $\varphi$ by an analogue of the classical Borel-Pompeiu formula, namely,

$$
\varphi \mid \Delta=\mathcal{C}_{\partial \Delta} \varphi+\mathcal{C}_{\Delta} \varphi
$$

It should be pointed out that $\mathcal{C}_{\partial \Delta} \varphi \in \mathfrak{M}(\Delta, \mathfrak{H})$ for any $\varphi \in C^{\infty}(\Omega, \mathfrak{H})$. Moreover, if $\varphi \in \mathfrak{M}(\Omega, \mathfrak{H})$, then $\varphi \mid \Delta=\mathcal{C}_{\partial \Delta} \varphi$. The second term $\mathcal{C}_{\Delta} \varphi$ in the right side of (2.8) is a bounded function on $\Delta$ for any $\varphi \in C^{\infty}(\Omega, \mathfrak{H})$. One of our main goals in what follows will be to find uniform estimates of $\mathcal{C}_{\Delta} \varphi$ in terms of $D \varphi \mid \Delta$.

2.4. More specifically, we are going to examine the convolution operator associated with the Cauchy kernel (2.5). In the sequel we will let $L^{1}\left(\mathbb{R}^{m+1}, \mathcal{H}\right)$ and $L^{\infty}\left(\mathbb{R}^{m+1}, \mathcal{H}\right)$ denote the usual Lebesgue spaces of integrable or essentially bounded $\mathcal{H}$-valued functions on $\mathbb{R}^{m+1}$, where either $\mathcal{H}=\mathbb{R}$, or $\mathcal{H}=\mathfrak{H}$ with $\mathfrak{H}$ an $\mathfrak{A}_{m}$-module as in 2.1. The norms on these spaces are denoted by $\|\cdot\|_{1}$ and $\|\cdot\|_{\infty}$, respectively. 
To every $\varphi \in L^{1}\left(\mathbb{R}^{m+1}, \mathcal{H}\right)$ we associate the function $E * \varphi$ on $\mathbb{R}^{m+1}$ defined by

$$
E * \varphi(a)=\frac{1}{\sigma_{m}} \int_{\mathbb{R}^{m+1}} \frac{\bar{x}}{|x|^{m+1}} \varphi(a-x) \mathrm{d} \lambda(x), \quad a \in \mathbb{R}^{m+1} .
$$

If $\mathcal{H}=\mathbb{R}$, then $E * \varphi$ is an $\mathbb{R}^{m+1}$-valued function. In case $\mathcal{H}=\mathfrak{H}$ we have an $\mathfrak{H}$-valued function. Under the additional assumption that $\varphi$ is essentially bounded we get that $E * \varphi$ is a bounded function. The precise result is stated below.

2.5. Theorem. Let $\mathbb{B}^{m+1}=\left\{x \in \mathbb{R}^{m+1}:|x| \leq 1\right\}$ and $\mathbb{K}^{m+1}=\left\{x \in \mathbb{R}^{m+1}\right.$ : $\left.|x|^{m+1} \leq x_{0}\right\}$.

(i) If $\varphi \in L^{1}\left(\mathbb{R}^{m+1}, \mathbb{R}\right) \cap L^{\infty}\left(\mathbb{R}^{m+1}, \mathbb{R}\right)$ and $\varphi \geq 0$, then

$$
|E * \varphi(a)| \leq \alpha_{m}^{+}\|\varphi\|_{1}^{1 /(m+1)}\|\varphi\|_{\infty}^{m /(m+1)}, \quad a \in \mathbb{R}^{m+1},
$$

where $\alpha_{m}^{+}=\left[\lambda\left(\mathbb{B}^{m+1}\right)\right]^{-1}\left[\lambda\left(\mathbb{K}^{m+1}\right)\right]^{m /(m+1)}$.

(ii) If $\varphi \in L^{1}\left(\mathbb{R}^{m+1}, \mathbb{R}\right) \cap L^{\infty}\left(\mathbb{R}^{m+1}, \mathbb{R}\right)$, then

$$
|E * \varphi(a)| \leq \alpha_{m}\|\varphi\|_{1}^{1 /(m+1)}\|\varphi\|_{\infty}^{m /(m+1)}, \quad a \in \mathbb{R}^{m+1},
$$

where $\alpha_{m}=2^{m /(m+1)} \alpha_{m}^{+}$.

(iii) If $\varphi \in L^{1}\left(\mathbb{R}^{m+1}, \mathfrak{H}\right) \cap L^{\infty}\left(\mathbb{R}^{m+1}, \mathfrak{H}\right)$, then

$$
\|E * \varphi(a)\|_{\mathfrak{H}} \leq \beta_{m}\|\varphi\|_{1}^{1 /(m+1)}\|\varphi\|_{\infty}^{m /(m+1)}, \quad a \in \mathbb{R}^{m+1},
$$

where $\|\cdot\|_{\mathfrak{H}}$ is the norm on $\mathfrak{H}$ and $\beta_{m}=\left[\lambda\left(\mathbb{B}^{m+1}\right)\right]^{-1 /(m+1)}$.

Moreover, the three inequalities (2.9), (2.10), and (2.11) above are in sharp form.

Proof. (i) Suppose that both $\varphi \in L^{1}\left(\mathbb{R}^{m+1}, \mathbb{R}\right) \cap L^{\infty}\left(\mathbb{R}^{m+1}, \mathbb{R}\right)$ and $a \in \mathbb{R}^{m+1}$ are fixed and let $A$ be an orthogonal transformation on $\mathbb{R}^{m+1}$. We notice that

$$
A(E * \varphi(a))=E * \varphi_{A, a}(0),
$$

where $\varphi_{A, a}(x)=\varphi\left(A^{-1} x+a\right), x \in \mathbb{R}^{m+1}$. Further, we choose $A$ so that

$$
A(E * \varphi(a))=-\mathcal{I} e_{0}, \quad \mathcal{I} \geq 0,
$$

where in fact

$$
\mathcal{I}=\frac{1}{\sigma_{m}} \int_{\mathbb{R}^{m+1}} \frac{x_{0}}{|x|^{m+1}} \varphi_{A, a}(x) \mathrm{d} \lambda(x) .
$$

Since $|E * \varphi(a)|=\mathcal{I}$ and $\|\varphi\|_{p}=\left\|\varphi_{A, a}\right\|_{p}$ for $p=1$ or $p=\infty$, we conclude that as far as inequality (2.9) is concerned, it would be enough to find upper estimates for

$$
\mathcal{I}(\varphi)=\frac{1}{\sigma_{m}} \int_{\mathbb{R}^{m+1}} \frac{x_{0}}{|x|^{m+1}} \varphi(x) \mathrm{d} \lambda(x)
$$

under the additional assumption $\mathcal{I}(\varphi) \geq 0$.

Suppose now that $\varphi$ is a non-negative function satisfying $\mathcal{I}(\varphi) \geq 0$, and let $\delta$ be a positive number. Set $K_{\delta}=\left\{x \in \mathbb{R}^{m+1}:|x|^{m+1} \leq \delta^{m} x_{0}\right\}$ and $K_{\delta}^{\mathrm{c}}=\mathbb{R}^{m+1} \backslash K_{\delta}$. From (2.15) we get

$$
\begin{aligned}
\sigma_{m} \mathcal{I}(\varphi) \leq & \int_{K_{\delta}}\left(\frac{x_{0}}{|x|^{m+1}}-\frac{1}{\delta^{m}}\right) \varphi(x) \mathrm{d} \lambda(x)+\frac{1}{\delta^{m}} \int_{K_{\delta}} \varphi(x) \mathrm{d} \lambda(x) \\
& +\int_{K_{\delta}^{\mathbb{C}}} \frac{x_{0}}{|x|^{m+1}} \varphi(x) \mathrm{d} \lambda(x) .
\end{aligned}
$$


Further, we notice that

$$
\int_{K_{\delta}}\left(\frac{x_{0}}{|x|^{m+1}}-\frac{1}{\delta^{m}}\right) \varphi(x) \mathrm{d} \lambda(x) \leq\|\varphi\|_{\infty} \int_{K_{\delta}}\left(\frac{x_{0}}{|x|^{m+1}}-\frac{1}{\delta^{m}}\right) \mathrm{d} \lambda(x) .
$$

We claim that

$$
\int_{K_{\delta}}\left(\frac{x_{0}}{|x|^{m+1}}-\frac{1}{\delta^{m}}\right) \mathrm{d} \lambda(x)=\frac{m}{\delta^{m}} \lambda\left(K_{\delta}\right) .
$$

To prove (2.18) we express any $x$ in $\mathbb{R}^{m+1}$ as $x=(\rho \cos \theta) e_{0}+(\rho \sin \theta) \omega$ with $\rho \geq 0,0 \leq \theta \leq \pi$, and $\omega$ a point on the unit sphere $\mathbb{S}^{m-1}$ in the $m$-dimensional subspace generated by $\left\{e_{1}, \ldots, e_{m}\right\}$. An easy calculation shows that $\mathrm{d} \lambda(x)=$ $\rho^{m}(\sin \theta)^{m-1} \mathrm{~d} \rho \mathrm{d} \theta \mathrm{d} \sigma(\omega)$, where $\sigma$ stands for the surface area measure on $\mathbb{S}^{m-1}$. Moreover, the compact set $K_{\delta}$ can be described in terms of $\rho, \theta$ and $\omega$ by

$$
0 \leq \theta \leq \frac{\pi}{2}, \quad 0 \leq \rho \leq \delta(\cos \theta)^{1 / m}, \quad \omega \in \mathbb{S}^{m-1} .
$$

Accordingly, we have

$$
\begin{aligned}
\int_{K_{\delta}} & \left(\frac{x_{0}}{|x|^{m+1}}-\frac{1}{\delta^{m}}\right) \mathrm{d} \lambda(x) \\
& =\int_{\mathbb{S}^{m-1}} \int_{0}^{\frac{\pi}{2}} \int_{0}^{\delta(\cos \theta)^{1 / m}}\left(\cos \theta-\frac{\rho^{m}}{\delta^{m}}\right)(\sin \theta)^{m-1} \mathrm{~d} \rho \mathrm{d} \theta \mathrm{d} \sigma(\omega) \\
& =\frac{\delta m}{m+1} \int_{\mathbb{S}^{m-1}} \int_{0}^{\frac{\pi}{2}}(\cos \theta)^{(m+1) / m}(\sin \theta)^{m-1} \mathrm{~d} \theta \mathrm{d} \sigma(\omega) .
\end{aligned}
$$

On the other hand we get

$$
\begin{aligned}
\lambda\left(K_{\delta}\right) & =\int_{\mathbb{S}^{m-1}} \int_{0}^{\frac{\pi}{2}} \int_{0}^{\delta(\cos \theta)^{1 / m}} \rho^{m}(\sin \theta)^{m-1} \mathrm{~d} \rho \mathrm{d} \theta \mathrm{d} \sigma(\omega) \\
& =\frac{\delta^{m+1}}{m+1} \int_{\mathbb{S}^{m-1}} \int_{0}^{\frac{\pi}{2}}(\cos \theta)^{(m+1) / m}(\sin \theta)^{m-1} \mathrm{~d} \theta \mathrm{d} \sigma(\omega),
\end{aligned}
$$

and so the proof of (2.18) is complete. By (2.17) and (2.18) it follows that

$$
\int_{K_{\delta}}\left(\frac{x_{0}}{|x|^{m+1}}-\frac{1}{\delta^{m}}\right) \varphi(x) \mathrm{d} \lambda(x) \leq \frac{m}{\delta^{m}} \lambda\left(K_{\delta}\right)\|\varphi\|_{\infty} .
$$

Another inequality we need is

$$
\int_{K_{\delta}^{\mathrm{c}}} \frac{x_{0}}{|x|^{m+1}} \varphi(x) \mathrm{d} \lambda(x) \leq \frac{1}{\delta^{m}} \int_{K_{\delta}^{\mathrm{c}}} \varphi(x) \mathrm{d} \lambda(x),
$$

which obviously follows from the definition of $K_{\delta}$. Finally, (2.16), (2.19) and (2.20) imply

$$
\sigma_{m} \mathcal{I}(\varphi) \leq \frac{m}{\delta^{m}} \lambda\left(K_{\delta}\right)\|\varphi\|_{\infty}+\frac{1}{\delta^{m}}\|\varphi\|_{1} .
$$

We next notice that $K_{\delta}=\delta \mathbb{K}^{m+1}$, hence $\lambda\left(K_{\delta}\right)=\delta^{m+1} \lambda\left(\mathbb{K}^{m+1}\right)$. On the other hand we have $\sigma_{m}=(m+1) \lambda\left(\mathbb{B}^{m+1}\right)$, so instead of (2.21) we may write

$$
\mathcal{I}(\varphi) \leq \frac{1}{(m+1) \lambda\left(\mathbb{B}^{m+1}\right)}\left[m \delta \lambda\left(\mathbb{K}^{m+1}\right)\|\varphi\|_{\infty}+\frac{1}{\delta^{m}}\|\varphi\|_{1}\right] .
$$


Now we take

$$
\delta=\left[\frac{\|\varphi\|_{1}}{\lambda\left(\mathbb{K}^{m+1}\right)\|\varphi\|_{\infty}}\right]^{1 /(m+1)} .
$$

For this specific value of $\delta$, inequality (2.22) becomes

$$
\mathcal{I}(\varphi) \leq\left[\lambda\left(\mathbb{B}^{m+1}\right)\right]^{-1}\left[\lambda\left(\mathbb{K}^{m+1}\right)\right]^{m /(m+1)}\|\varphi\|_{1}^{1 /(m+1)}\|\varphi\|_{\infty}^{m /(m+1)} .
$$

The proof of assertion (i) is complete.

(ii) We assume that $\varphi$ is a real-valued function, and let $\varphi=\varphi_{+}-\varphi_{-}$be its Jordan decomposition. We clearly have

$$
|E * \varphi(a)| \leq\left|E * \varphi_{+}(a)\right|+\left|E * \varphi_{-}(a)\right|, \quad a \in \mathbb{R}^{m+1} .
$$

Inequality (2.9) applied to $\varphi_{+}$and $\varphi_{-}$implies

$$
\begin{aligned}
|E * \varphi(a)| & \leq \alpha_{m}^{+}\left[\left\|\varphi_{+}\right\|_{1}^{1 /(m+1)}\left\|_{\varphi_{+}}\right\|_{\infty}^{m /(m+1)}\right. \\
& \left.+\left\|\varphi_{-}\right\|_{1}^{1 /(m+1)}\left\|\varphi_{-}\right\|_{\infty}^{m /(m+1)}\right], \quad a \in \mathbb{R}^{m+1}
\end{aligned}
$$

On the other hand $\left\|\varphi_{ \pm}\right\|_{\infty} \leq\|\varphi\|_{\infty}$, and

$$
\begin{aligned}
\frac{1}{2}\left[\left\|\varphi_{+}\right\|_{1}^{1 /(m+1)}+\left\|\varphi_{-}\right\|_{1}^{1 /(m+1)}\right] & \leq\left[\frac{\left\|\varphi_{+}\right\|_{1}+\left\|\varphi_{-}\right\|_{1}}{2}\right]^{1 /(m+1)} \\
& =2^{-1 /(m+1)}\|\varphi\|_{1}^{1 /(m+1)} .
\end{aligned}
$$

Therefore,

$$
|E * \varphi(a)| \leq 2^{m /(m+1)} \alpha_{m}^{+}\|\varphi\|_{1}^{1 /(m+1)}\|\varphi\|_{\infty}^{m /(m+1)}, \quad a \in \mathbb{R}^{m+1}
$$

The proof of inequality (2.10) is complete.

(iii) We first observe that

$$
|E(x)|=\frac{1}{\sigma_{m}} \cdot \frac{1}{|x|^{m}}, \quad x \in \mathbb{R}_{0}^{m+1}
$$

Therefore, given $\varphi$ an $\mathfrak{H}$-valued function and $a \in \mathbb{R}^{m+1}$ we have

$$
\|E * \varphi(a)\|_{\mathfrak{H}} \leq \frac{1}{\sigma_{m}} \int_{\mathbb{R}^{m+1}} \frac{\varphi_{a}(x)}{|x|^{m}} \mathrm{~d} \lambda(x),
$$

where $\varphi_{a}(x)=\|\varphi(a-x)\|_{\mathfrak{H}}, x \in \mathbb{R}^{m+1}$. Since $\|\varphi\|_{p}=\left\|\varphi_{a}\right\|_{p}$ for $p=1$ or $p=\infty$, it would suffice to find on upper bound for the integral

$$
\mathcal{J}(\varphi)=\frac{1}{\sigma_{m}} \int_{\mathbb{R}^{m+1}} \frac{\varphi(x)}{|x|^{m}} \mathrm{~d} \lambda(x)
$$

where now $\varphi$ stands for a non-negative function.

To this end we repeat the proof of assertion (i) using $\mathbb{B}^{m+1}$ instead of $\mathbb{K}^{m+1}$. For the sake of convenience we mention that inequality (2.22) is replaced by

$$
\mathcal{J}(\varphi) \leq \frac{1}{(m+1) \lambda\left(\mathbb{B}^{m+1}\right)}\left[m \delta \lambda\left(\mathbb{B}^{m+1}\right)\|\varphi\|_{\infty}+\frac{1}{\delta^{m}}\|\varphi\|_{1}\right],
$$

and we may take

$$
\delta=\left[\frac{\|\varphi\|_{1}}{\lambda\left(\mathbb{B}^{m+1}\right)\|\varphi\|_{\infty}}\right]^{1 /(m+1)}
$$


In effect we get

$$
\mathcal{J}(\varphi) \leq\left[\lambda\left(\mathbb{B}^{m+1}\right)\right]^{-1(m+1)}\|\varphi\|_{1}^{1 /(m+1)}\|\varphi\|_{\infty}^{m /(m+1)},
$$

an inequality that implies (2.11).

In order to show that $(2.9),(2.10)$, and (2.11) are sharp we indicate in each case an extreme function. To begin with, we claim that the function $\varphi$ defined as

$$
\varphi(x)= \begin{cases}1, & \text { if }-x \in \mathbb{K}^{m+1}, \\ 0, & \text { otherwise }\end{cases}
$$

is an extreme function for (2.9). We first notice that

$$
|E * \varphi(0)|=\frac{1}{\sigma_{m}} \int_{\mathbb{K}^{m+1}} \frac{x_{0}}{|x|^{m+1}} \mathrm{~d} \lambda(x)=\frac{m+1}{\sigma_{m}} \lambda\left(\mathbb{K}^{m+1}\right),
$$

the last equality being a consequence of (2.18). On the other hand, the right side of $(2.9)$ is given by

$$
\alpha_{m}^{+}\|\varphi\|_{1}^{1 /(m+1)} \|_{\infty}^{m /(m+1)}=\left[\lambda\left(\mathbb{B}^{m+1}\right)\right]^{-1} \lambda\left(\mathbb{K}^{m+1}\right)=\frac{m+1}{\sigma_{m}} \lambda\left(\mathbb{K}^{m+1}\right) .
$$

In a similar way we establish that the real-valued function $\varphi$ defined as

$$
\varphi(x)= \begin{cases}1, & \text { if }-x \in \mathbb{K}^{m+1}, \\ -1, & \text { if } x \in \mathbb{K}^{m+1}, \\ 0, & \text { otherwise, }\end{cases}
$$

is an extreme function for (2.10). Finally, let us take the $\mathfrak{A}_{m}$-valued function $\varphi$ defined as

$$
\varphi(x)= \begin{cases}x /|x|, & \text { if } x \in \mathbb{B}^{m+1} \backslash\{0\}, \\ 0, & \text { otherwise. }\end{cases}
$$

Since $\bar{x} \cdot x=|x|^{2}$ for any $x \in \mathbb{R}^{m+1}$, we easily get $\|E * \varphi(0)\|_{\mathfrak{A}_{m}}=1$. In addition, $\|\varphi\|_{1}=\lambda\left(\mathbb{B}^{m+1}\right)$ and $\|\varphi\|_{\infty}=1$, hence $\varphi$ is an extreme function for (2.11).

2.6. We conclude this section with a few remarks. First we mention that when $\varphi$ is the characteristic function of a compact set $X$ in $\mathbb{R}^{m+1}$ we get

$$
\left|\frac{1}{\sigma_{m}} \int_{X} \frac{\bar{a}-\bar{x}}{|a-x|^{m+1}} \mathrm{~d} \lambda(x)\right| \leq \alpha_{m}^{+}[\lambda(X)]^{1 /(m+1)}, \quad a \in \mathbb{R}^{m+1}
$$

an inequality that provides the announced higher-dimensional version of (1.1).

Another point we want to make is that the reasonings developed above essentially rely upon some specific features of the Cauchy kernel $E$. A slight variation is readily at hand. We replace $E$ by the kernel

$$
E_{A, k}=\frac{1}{\sigma_{m}} \cdot \frac{A x}{|x|^{k+1}}, \quad x \in \mathbb{R}_{0}^{m+1}
$$

where $A$ is an orthogonal transformation on $\mathbb{R}^{m+1}$ and $0<k<m+1$. Theorem 2.5 needs a few minor adjustments. First, instead of $\mathbb{K}^{m+1}$ we take the compact set

$$
\mathbb{K}_{k}^{m+1}=\left\{x \in \mathbb{R}^{m+1}:|x|^{k+1} \leq x_{0}\right\}
$$


Moreover, instead of $\alpha_{m}^{+}, \alpha_{m}$, and $\beta_{m}$ we need the constants

$$
\begin{gathered}
\alpha_{m, k}^{+}=\left[(m+1-k) \lambda\left(\mathbb{B}^{m+1}\right)\right]^{-1}\left[\lambda\left(\mathbb{K}_{k}^{m+1}\right)\right]^{k /(m+1)}, \\
\alpha_{m, k}=2^{m /(m+1)} \alpha_{m, k}^{+}, \\
\beta_{m, k}=(m+1-k)^{-1}\left[\lambda\left(\mathbb{B}^{m+1}\right)\right]^{-(m+1-k) /(m+1)}
\end{gathered}
$$

Other estimates similar to (2.9), (2.11), and (2.13) in Theorem 2.5 for a different kind of kernels have been established in $[\mathrm{K}]$ and $[\mathrm{GK}]$. A more general situation is investigated in $[\mathrm{MSz}]$.

\section{A generalization of Alexander's inequality}

Our next goal is to derive higher-dimensional analogues of Alexander's inequality (1.2). As a substitute for rational functions on $\mathbb{C}$ we will employ Clifford analytic functions defined on open subsets of $\mathbb{R}^{m+1}$ and with values in a Clifford module.

3.1. Besides the notations introduced before, we let $C(X, \mathfrak{H})$ denote the Banach space of all $\mathfrak{H}$-valued continuous functions on a compact set $X \subset \mathbb{R}^{m+1}$. The norm on $C(X, \mathfrak{H})$ is given by

$$
\|\varphi\|_{\infty, X}=\sup _{x \in X}\|\varphi(x)\|_{\mathfrak{H}}, \quad \varphi \in C(X, \mathfrak{H}),
$$

where $\|\cdot\|_{\mathfrak{H}}$ is the norm on the Hilbert space $\mathfrak{H}$. In addition, we consider the subspace $\mathfrak{M}(X, \mathfrak{H})$ of $C(X, \mathfrak{H})$ consisting of restrictions to $X$ of functions from $\mathfrak{M}(\Omega, \mathfrak{H})$, where $\Omega$ is an open neighborhood of $X$ in $\mathbb{R}^{m+1}$.

The next result and its corollary might be thought of as a straightforward generalization of Alexander's inequality (1.2).

Proposition. Suppose $\varphi \in C(X, \mathfrak{H})$ is the restriction to $X$ of a linear function $\varphi: \mathbb{R}^{m+1} \rightarrow \mathfrak{H}$. Then

$$
\operatorname{dist}_{C(X, \mathfrak{H})}[\varphi, \mathfrak{M}(X, \mathfrak{H})] \leq \alpha_{m}^{+}[\lambda(X)]^{1 /(m+1)}\|D \varphi\|_{\infty, X} .
$$

Proof. Let $\Delta$ be a bounded open neighborhood of $X$ in $\mathbb{R}^{m+1}$ with a smooth boundary. By Borel-Pompeiu formula (2.8) we easily get

$$
\operatorname{dist}_{C(X, \mathfrak{H})}[\varphi, \mathfrak{M}(X, \mathfrak{H})] \leq\left\|\mathcal{C}_{\Delta} \varphi\right\|_{\infty, X} .
$$

On the other hand, there exists a vector $\xi \in \mathfrak{H}$ such that $D \varphi(x)=\xi$ for any point $x \in \Delta$. By (2.7) we deduce that

$$
\mathcal{C}_{\Delta} \varphi(a)=E * \chi_{\bar{\Delta}}(a) \xi, \quad a \in X
$$

where $\chi_{\bar{\Delta}}$ is the characteristic function of the closure of $\Delta$ in $\mathbb{R}^{m+1}$. Therefore,

$$
\left\|\mathcal{C}_{\Delta} \varphi\right\|_{\infty, X}=\sup _{a \in X}\left|E * \chi_{\bar{\Delta}}(a)\right|\|\xi\|_{\mathfrak{H}} .
$$

By using now the Ahlfors-Beurling type inequality (2.30), from (3.5) we deduce that

$$
\left\|\mathcal{C}_{\Delta} \varphi\right\|_{\infty, X} \leq \alpha_{m}^{+}[\lambda(\bar{\Delta})]^{1 /(m+1)}\|\xi\|_{\mathfrak{H}}
$$

Inequality (3.1) follows from (3.3) and (3.6) by letting $\Delta$ approach $X$ and observing that $\|D \varphi\|_{\infty, X}=\|\xi\|_{\mathfrak{H}}$.

In case $\mathfrak{H}=\mathfrak{A}_{m}$ and $\varphi(x)=\bar{x}, x \in \mathbb{R}^{m+1}$, we get the next direct extension of Alexander's inequality. 
3.5. Corollary. Considering $\bar{x}$ as a function in $C\left(X, \mathfrak{A}_{m}\right)$ we have

$$
\operatorname{dist}_{C\left(X, \mathfrak{A}_{m}\right)}\left[\bar{x}, \mathfrak{M}\left(X, \mathfrak{A}_{m}\right)\right] \leq(m+1)^{1 / 2} \alpha_{m}^{+}[\lambda(X)]^{1 /(m+1)} .
$$

Unfortunately, the Stone-Weierstrass Theorem and inequality (3.7) do not suffice to conclude that $\mathfrak{M}\left(X, \mathfrak{A}_{m}\right)$ is a dense subspace of $C\left(X, \mathfrak{A}_{m}\right)$ when $X$ has Lebesgue measure zero. A possible remedy is indicated below.

3.3. In the same setting as above, we let $C^{\infty}(X, \mathfrak{H})$ denote the space consisting of restrictions to $X$ of smooth $\mathfrak{H}$-valued functions defined on open neighborhoods of $X$ in $\mathbb{R}^{m+1}$. By the Stone-Weierstrass Theorem we get that $C^{\infty}(X, \mathfrak{H})$ is a dense subspace of $C(X, \mathfrak{H})$. The next result can be proved by employing the AhlforsBeurling type inequality (2.11) together with a reasoning similar to that developed in the proof of Proposition 3.1.

Proposition. If $\varphi \in C^{\infty}(X, \mathfrak{H})$, then

$$
\operatorname{dist}_{C(X, \mathfrak{H})}[\varphi, \mathfrak{M}(X, \mathfrak{H})] \leq \beta_{m}[\lambda(X)]^{1 /(m+1)}\|D \varphi\|_{\infty, X} .
$$

3.7. Corollary (Hartogs-Rosenthal). Let $X$ be a compact subset of $\mathbb{R}^{m+1}$ with Lebesgue measure zero. Then $\mathfrak{M}(X, \mathfrak{H})$ is a dense subspace of $C(X, \mathfrak{H})$.

For a different proof of the multi-dimensional Hartogs-Rosenthal theorem stated above we refer to [BDS, Section 18].

\section{REFERENCES}

[AB] L. Ahlfors, and A. Buerling, Conformal invariants and function theoretic null sets, Acta Math. 83 (1950), 101-129. MR 12:171e

[A1] H. Alexander, Projections of polynomial hulls, J. Funct. Anal. 13 (1973), 13-19. MR 49:3209

[A2] H. Alexander, On the area of the spectrum of an element of a uniform algebra, Complex Approximation Proceedings, Quebec, July 3-8, 1978, Birkhäuser, 1980, pp. 3-12. MR 82b:32015

[AS] S. Axler, and J. H. Shapiro, Putnam's theorem, Alexander's spectral area estimate, and VMO, Math. Ann. 271 (1985), 161-183. MR 87b:30053

[B] A. Browder, Introduction to Function Algebras, Benjamin, New York, 1969. MR 39:7431

[BDS] F. Brackx, R. Delanghe, and F. Sommen, Clifford Analysis, Pitman Research Notes in Mathematics Series, 76, 1982. MR 85j:30103

[DSS] R. Delanghe, F. Sommen, and V. Souček, Clifford Algebra and Spinor-Valued Functions, Kluwer Academic Publishers, 1992. MR 94d:30084

[G] T. W. Gamelin, Uniform Algebras, Prentice Hall, 1969. MR 53:14137

[GM] J. E. Gilbert and M. A. M. Murray, Clifford Algebras and Dirac Operators in Harmonic Analysis, Cambridge Studies in Advanced Mathematics, 26, Cambridge University Press, 1991. MR 93e: 42027

[GK] B. Gustafsson and D. Khavinson, On approximation by harmonic vector fields, Houston J. Math. 20 (1994), 75-92. MR 96h:31009

[K] D. Khavinson, On uniform approximation by harmonic functions, Mich. Math. J. 34 (1987), 465-473. MR 89a:41026

[MSz] M. Martin, and P. Szeptycki, Sharp inequalities for convolution operators with homogeneous kernels and applications, Indiana Univ. Math. J. 46 (1997).

[P] M. Putinar, Extreme hyponormal operators, Operator Theory: Advances and Applications, 28, 1988, pp. 249-265. MR 90a:47065

$[\mathrm{Pu}]$ C. R. Putnam, An inequality for the area of hyponormal spectra, Math. Z. 116 (1970), 323-330. MR 42:5085

[VPY] A. L. Volberg, V. V. Peller, and D. V. Yakubovich, A brief excursion into the theory of hyponormal operators, Algebra i Analiz 2 (1990), 1-30. MR 91i:47034

Department of Mathematics, Baker University, Baldwin City, Kansas 66006

E-mail address: mmartin@harvey.bakeru.edu 